\title{
POLÍTICAS DE INCLUSIÓN EDUCATIVA DE ADOLESCENTES, JÓVENES Y ADULTOS EN LA PROVINCIA DE BUENOS AIRES: TENSIONES EN TORNO A LA SEGMENTACIÓN SOCIO EDUCATIVA Y EL ACCESO AL CONOCIMIENTO
}

\author{
Jorgelina Silvia Sassera* \\ CONICET- Universidad de Buenos Aires, Argentina \\ jsassera@filo.uba.ar \\ Natalia Herger ** \\ Universidad de Buenos Aires, Argentina \\ nath@filo.uba.ar
}

Recibido: 15/06/2020 - Aceptado: 14/11/2020

\section{Resumen}

Las desigualdades en la atención del derecho a la educación se asientan en las desigualdades estructurales que tienen incidencia en la configuración y dinámica de los sistemas educativos. La expansión de un nivel educativo a nuevos sectores sociales se produce a la par de una segmentación que incidiría en el acceso igualitario al conocimiento. En el marco de la obligatoriedad de la educación secundaria y del esfuerzo ampliar la escolarización, se formularon iniciativas y experiencias que se proponen incluir y acompañar a los jóvenes y adultos que se encontraban por fuera del sistema. Este artículo explora las condiciones de acceso al conocimiento en el Plan de Finalización de Estudios Primarios y Secundarios (FinES) en la provincia de Buenos Aires desde su creación y los cambios ocurridos a partir de 2016 mediante el análisis de la organización del plan de estudios, las formas de dictado de las clases, las condiciones de cursada y la forma de incorporación de los docentes. Un interrogante es si estas políticas contribuirían a formar circuitos de baja intensidad institucional, y los desafíos para garantizar la apropiación de saberes socialmente relevantes o valorados con niveles de calidad equivalentes a los del resto del sistema.

Palabras clave: Políticas educativas - Segmentación socio-educativa - Conocimiento Curriculum - Educación de adolescentes - Jóvenes y adultos.

\footnotetext{
Tnvestigadora Asistente CONICET con sede en el Programa Educación, Economía y Trabajo del Instituto de Investigaciones en Ciencias de la Educación, Facultad de Filosofía y Letras de la Universidad de Buenos Aires (PEETIICE-FFyL/UBA) bajo la dirección de la Dra. Graciela C. Riquelme. Doctora de la Universidad de Buenos Aires en Ciencias de la Educación, Magíster en Ciencias Sociales con orientación en Educación (FLACSO). Licenciada en Sociología (UBA). Profesora adjunta interina de Perspectivas Comparadas de Políticas Públicas Educativas (FCEDU-UNER). * Investigadora del Programa Educación. Economía y Trabajo del Instituto de Investigaciones en Ciencias de la Educación, Facultad de Filosofía y Letras de la Universidad de Buenos Aires (PEET-IICE-FFyL/UBA). Doctora de la Universidad de Buenos Aires en Ciencias de la Educación. Magíster en Diseño y Gestión de Programas Sociales (FLACSO). Licenciada en Ciencias de la Educación (UBA). Profesora adjunta interina de Economía Política de la Educación en la Licenciatura en Ciencias de la Educación (UBA).
} 


\title{
EDUCATIONAL INCLUSION OF ADOLESCENTS, YOUTH AND ADULTS POLICIES IN THE PROVINCE OF BUENOS AIRES: TENSIONS AROUND SOCIO- EDUCATIONAL SEGMENTATION AND ACCESS TO KNOWLEDGE
}

\begin{abstract}
The structural inequalities have an impact on the attention to the right to education and on the configuration and dynamics of the educational systems. The expansion of an educational level occurs along with a segmentation that would affect equal access to knowledge. In the framework of compulsory secondary education and the effort to expand schooling, there are initiatives and experiences that aim to include young people and adults who are outside the system. This article explores the conditions of access to knowledge in the Plan of Completion of Primary and Secondary Studies (FinES) in the province of Buenos Aires since its creation to the changes that have occurred since 2016. The article explores the organization of the study plan, the classes' organization, and study and teachers work conditions. A question is whether these policies contribute to create low institutional intensity circuits, and which are the challenges regarding socially relevant knowledge appropriation with quality levels equivalent to those of the rest of the system.
\end{abstract}

Keywords: Educational policies - Socio-educational segmentation - Knowledge - Curriculum - Youth and adults education.

\section{Introducción}

El derecho a la educación fue un postulado que orientó la política educativa de Argentina desde mediados de la década del 2000, expresado en la obligatoriedad del nivel secundario y la implementación de programas dirigidos a favorecer el acceso, permanencia y la vuelta al sistema educativo para adolescentes, jóvenes y adultos. Estas medidas pueden plantear tensiones y contradicciones en el marco de la segmentación del sistema educativo y en particular de la educación secundaria, pues si bien promovieron la inclusión educativa de jóvenes y adultos, surge el interrogante sobre si lo hacen en trayectos o circuitos educativos con algunas características de fragilidad institucional.

En Argentina mucho se ha estudiado acerca de las desigualdades en el acceso y permanencia de la población en el sistema educativo, dando cuenta que la intención universalista que primó en su construcción no logró concretarse, y que persisten grupos que no han asistido o no han alcanzado la educación obligatoria (Riquelme, 1978 y 2000 y Riquelme y Herger, 2005, Riquelme \& Kodric, 2013). Ello ha sido interpretado como "distribución ilusoria" ya que, pese a la expansión del sistema, se mantiene la desigualdad entre estratos socioeconómicos (Riquelme, Herger y Sassera, 2018, a y b).

Las desigualdades en la atención del derecho a la educación se asientan en las desigualdades estructurales que tienen incidencia en la configuración y dinámica de los sistemas educativos. La expansión de un nivel educativo a nuevos sectores sociales suele producirse a la par de una segmentación que los orientaría hacia determinados trayectos o carriles que en muchos casos han perdido valor para la movilidad social. Un aspecto a tener en cuenta para comprender la segmentación socioeducativa, remite a los saberes y conocimientos a los que acceden y se apropian los adolescentes, jóvenes y adultos de distintos sectores sociales que circulan por cada uno de estos trayectos.

Los aportes de Michael Young permiten reflexionar sobre conocimiento al que se accede en las instituciones escolares. Young (2009) da prioridad al conocimiento que se transmite en los planes de estudio y valoriza el conocimiento poderoso, es decir aquel transmitido mediante el curriculum escolar basado en las disciplinas científicas y que provee de explicaciones confiables y de nuevas formas de pensar sobre el mundo. El autor pone el acento sobre las políticas educativas, y puntualiza que no es lo mismo el acceso a los niveles educativos, que el acceso y apropiación del conocimiento que se transmite, especialmente cuando está estratificado (Young \& Muller, 2016).

El artículo, realizado en el marco de una investigación en curso $^{1}$, explora algunos aspectos acerca de las condiciones de acceso al conocimiento en un programa dirigido a que jóvenes y adultos completen la educación obligatoria, el Plan de Finalización de Estudios Primarios y Secundarios (FinES).

El artículo analiza la organización del plan de estudios, las formas de dictado de las clases, las condiciones de cursada y las condiciones de incorporación de los docentes tutores en la provincia de Buenos Aires a partir de fuentes secundarias. En este análisis, se recuperan aspectos de entrevistas

\footnotetext{
${ }^{1}$ Proyecto "Transformación de la educación secundaria y formación para el trabajo de la Argentina en las últimas décadas: Transiciones críticas y diferenciación provincial y territorial. Áreas de vacancia, desafíos e innovación" (PIP 2017-2019 / UBACyT 2018), dirigido por la Dra. Graciela Clotilde Riquelme con sede en el Programa Educación, Economía y Trabajo (PEET-IICE-FFyL/UBA). Página web: https://educacion-economia-trabajo-peet.org.
} 
REVISTA DE LA ESCUELA DE CIENCIAS DE LA EDUCACIÓN, AÑO 17, NRO. 16, VOL. 2, JULIO A DICIEMBRE DE 2021. PÁGINAS 13-25. ISSN 2362-3349 (EN LÍNEA). POLITICAS DE INCLUSIÓN EDUCATIVA DE ADOLESCENTES, JÓVENES Y ADULTOS EN LA PROVINCIA DE BUENOS AIRES: TENSIONES EN TORNO A LA SEGMENTACIÓN SOCIO EDUCATIVA Y EL ACCESO AL CONOCIMIENTO. JORGELINA SILVIA SASSERA. NATALIA HERGER

realizadas a directores, docentes, estudiantes de escuelas secundarias de jóvenes y adultos y de inspectores y coordinadores de programas de terminalidad educativa de las localidades de Campana, Zárate, Florencio Varela y Berazategui². También se realizó el análisis de normativas, diseños curriculares, documentos y de fuentes bibliográficas que permitieron caracterizar los cambios recientes registrados en FinEs.

El primer apartado presenta el tratamiento de la segmentación socio-educativa a partir de algunas investigaciones latinoamericanas y de Argentina. El segundo punto busca reflexionar sobre el acceso al conocimiento a partir de los aportes de M. Young y la noción de conocimiento poderoso y desde las perspectivas de otros autores sobre la justicia curricular. El último apartado explora las políticas de inclusión educativa de jóvenes y adultos y se interroga sobre las condiciones de acceso al conocimiento en el Plan Fines de la Provincia de Buenos Aires, indagando sobre algunas características de la organización institucional y curricular de este plan en el período reciente.

\section{La noción de segmentación socio educativa para comprender las desigualdades educativas socialmente esctructuradas}

La desigualdad educativa es un fenómeno complejo en el que intervienen factores múltiples, pero es preciso reconocer que existe un vínculo directo con la desigualdad de la estructura social, que se asienta en la heterogeneidad económica y productiva y da lugar a la segmentación del mercado de trabajo, de los ingresos y de la seguridad social, y de las condiciones de vida de la población. Existe una articulación compleja entre las desigualdades derivadas de la segmentación socioeconómica y la concreción del derecho a la educación de la población dando lugar a la diferenciación social en el acceso, permanencia y terminalidad de la educación secundaria.

En América Latina y en Argentina se realizaron aproximaciones al estudio sobre la segmentación socioeducativa, que presentaron limitaciones dado el restringido acceso a fuentes de información sociodemográfica adecuadas. Estos estudios clásicos indagaron en la relación entre sistemas educativos y su segmentación en las ramas técnicas y profesionales y los procesos de industrialización y segmentación del mercado de trabajo (Rama, 1977; Tedesco, 1977; Wiñar, 1981). En este marco, Riquelme (1985) resalta la heterogeneidad del sistema educativo y "una segmentación en las oportunidades de acceso y de permanencia en las instituciones (escuelas, centros y Universidades) por parte de los distintos grupos sociales" (Riquelme, 1985, p.18)

Otros trabajos se enfocaron sobre las formas de discriminación educativa, y analizaron los factores y dinámicas que producen a la segmentación educativa. Braslavsky (1985) identificó la dimensión horizontal, que remite a las similitudes y diferencias entre las oportunidades educativas de un mismo nivel del sistema de educación formal materializadas en escuelas donde se ofrece un curriculum diferente y que tienen además distintas condiciones para el aprendizaje.

A lo largo de las últimas décadas, numerosas investigaciones han intentado avanzar en el análisis de la segmentación socio-educativa y de procesos relacionados como la diferenciación institucional, la fragmentación y la segregación educativas. A fines de la década de los '90 y principios de los dos mil, una serie de estudios abordaron la segmentación del sistema desde distintas interpretaciones ante las evidencias de la profundización de las desigualdades educativas y la conformación de segmentos o circuitos (Riquelme, Herger \& Sassera, 2018a; Tedesco, 2001; Filmus et. al., 2001; Llach \& Schumacher, 2006).

Otras investigaciones del siglo XXI pusieron el acento en la existencia de una 'reproducción imperfecta' ante el aumento de la escolaridad de la población y la autonomía del sistema educativo (Filmus et. al. 2001) y sobre los cambios sociales y culturales y su incidencia en la configuración de un nivel secundario segmentado que conduce a una diferenciación de la experiencia escolar para los estudiantes (Kessler, 2002; Gallart, 2006; Dussel 2007; Tuñón \& Halperin 2009).

Un abordaje que aporta a la comprensión de la segmentación socio educativa, la interpreta como la existencia de circuitos, caminos o trayectos al interior de un mismo nivel que promueven trayectorias educativas desiguales para determinados grupos de la población; en términos de las características de la oferta. Ello se manifiesta en las divisiones entre lo público y lo privado, pero también al interior de cada uno respecto a la dotación diferencial de recursos, las características y perfiles institucionales y los conocimientos que se transmiten y aprenden en relación con la estructura social (Riquelme, Herger y Sassera, 2018a).

Investigaciones recientes, han permitido reconstruir grupos o circuitos educativos de escuelas secundarias, secundarias técnicas y agrarias de escuelas de jóvenes y adultos y otras instituciones de educación y formación para el trabajo en localidades de las provincias de Buenos Aires y Santa Fe (Riquelme, Herger \& Sassera, 2018a; Sassera, 2014).

El análisis de entrevistas realizadas a directivos de las escuelas secundarias y secundarias técnicas, escuelas de jóvenes y adultos y a coordinadores e inspectores de FinEs y otras políticas y experiencias de terminalidad educativa, permitió indagar cómo se diferencian las instituciones en cuanto a la localización de las mismas en el territorio de cada ciudad, las barreras que enfrenta la población para el acceso, las condiciones edilicias, la disponibilidad de recursos materiales adecuados y la caracterización ilustrativa del

\footnotetext{
${ }^{2}$ El trabajo de campo se desarrolló en distintas etapas entre los años 2013 y 2015, y la muestra total de escuelas
} secundarias fue de 56 instituciones y de 17 escuelas primarias y secundarias de jóvenes y adultos. 
REVISTA DE LA ESCUELA DE CIENCIAS DE LA EDUCACIÓN, AÑO 17, NRO. 16, VOL. 2, JULIO A DICIEMBRE DE 2021. PÁGINAS 13-25. ISSN 2362-3349 (EN LÍNEA). POLITICAS DE INCLUSIÓN EDUCATIVA DE ADOLESCENTES, JÓVENES Y ADULTOS EN LA PROVINCIA DE BUENOS AIRES: TENSIONES EN TORNO A LA SEGMENTACIÓN SOCIO EDUCATIVA Y EL ACCESO AL CONOCIMIENTO. JORGELINA SILVIA SASSERA. NATALIA HERGER

perfil de la matrícula. En el caso de las políticas de terminalidad se indagaron cuestiones referidas a los modos en los que se implementa el programa en los distritos y los desafíos enfrentados; la coordinación entre distintas áreas; y las características y recursos (financieros, docentes, de infraestructura, de gestión; etc.). Si bien en cada localidad y provincia los circuitos presentan características específicas, pueden sintetizarse de esta manera:

Tabla 1. Síntesis de características de los circuitos educativos

\begin{tabular}{|c|c|}
\hline Grupo I & $\begin{array}{l}\text { - Instituciones educativas prestigiosas, de localización predominantemente céntrica; } \\
\text { - edificios en condiciones adecuadas y recursos suficientes; } \\
\text { - relaciones con diversos actores de la realidad social y productiva; } \\
\text {-acumulación de recursos; } \\
\text { - - actividades pedagógicas para los estudiantes. }\end{array}$ \\
\hline Grupo II & $\begin{array}{l}\text { - Instituciones en situación intermedia, } \\
\text { - dificultad en el mantenimiento de los edificios; } \\
\text { - afectadas por barreras a la accesibilidad; } \\
\text { - instituciones de educación técnico-profesional. } \\
\text { - matrícula en crecimiento. }\end{array}$ \\
\hline Grupo III & $\begin{array}{l}\text { - Sumatoria de desigualdades múltiples; } \\
\text { - estudiantes que provienen de zonas con NBI moderado-alto; } \\
\text { - desfavorabilidad en la localización; } \\
\text { - barreras físicas y económicas; } \\
\text { - necesidad de más personal docente }\end{array}$ \\
\hline Grupo IV & $\begin{array}{l}\text { - Fragilidad institucional; } \\
\text { - propuestas de programas o planes con propuestas transitorias o "a renovación"; } \\
\text { - escasos recursos financieros y materiales; } \\
\text { - falta de planificación; } \\
\text { - atención de población con bajo nivel educativo. }\end{array}$ \\
\hline
\end{tabular}

Fuente: elaborado para este artículo a partir de Autor, Autor \& Autor (2018) y Autor (2018)

Los grupos de escuelas reconstruidos dan cuenta de instituciones que guardan similitudes entre sí, pero que presentan diferencias respecto a las de los demás grupos. El grupo I se compone por instituciones "prestigiosas", localizadas en el centro privilegiante de las ciudades estudiadas y que son elegidas por estudiantes de distinta procedencia social. El grupo II está integrado por escuelas que no presentan problemáticas profundas en su funcionamiento, pero que requerirían de mejoras edilicias y de las condiciones de accesibilidad a las mismas. El grupo III presenta las situaciones más desventajosas, se trata de establecimientos con necesidades edilicias, de recursos y que se localizan en barrios o zonas desfavorables de las localidades.

El grupo IV está conformado por programas de inclusión y terminalidad educativa para la población con bajo nivel educativo y que operan en condiciones de fragilidad institucional. Estas propuestas se formularon como experiencias a término pero se extendieron en el tiempo; cuentan con recursos limitados, los docentes trabajan en forma precaria y con contrato a término y los recursos edilicios y materiales son escasos Otros aspectos de la fragilidad institucional se manifiestan, en el desconocimiento sobre la operatoria de la política por parte de los múltiples actores que intervienen, en la incertidumbre que rodea su continuidad y la debilidad de las instancias de supervisión y seguimiento pedagógico (Riquelme, Herger \& Sassera, 2018a)

Reflexiones sobre el acceso al conocimiento en contextos de segmentación socio educativa

La distribución y apropiación desigual del conocimiento socialmente relevante entre los sectores sociales constituyen una expresión de la segmentación del sistema educativo, que se manifiesta en las diferencias en el acceso y la progresión entre los niveles, así como entre las distintas orientaciones, trayectos y circuitos en el mismo nivel. De esta manera la selección y organización de los contenidos que se incluyen en el curriculum de las distintas ofertas se correspondería con la segmentación de la estructura social, que diversifica las trayectorias educativas de los estudiantes de distintas clases sociales y limita el acceso igualitario al conocimiento para toda la población. La preocupación por cómo se selecciona, distribuye y organiza el conocimiento socialmente valorado resultaría central para garantizar el derecho de toda la población a estos saberes.

Young (2009) sostiene la importancia de la noción de conocimiento poderoso, el cual solo podría transmitirse en las instituciones educativas pues es institucionalizado y legítimo, y provee de explicaciones confiables y de nuevas formas de pensar sobre el mundo. Los contenidos curriculares son el conocimiento poderoso, de gran alcance, producido en los campos disciplinares y por ello fiable. El conocimiento poderoso se distancia del conocimiento cotidiano o conocimiento de la experiencia, accesible en múltiples ámbitos o adquirido en tareas específicas para realizar tareas específicas (Young \& Muller, 2013). En tanto el conocimiento teórico es el socialmente validado y una base para adquirir nuevo conocimiento, quienes no acceden a ellos tienen una posición desventajosa en la sociedad y dificultades para superar y profundizar los conocimientos más allá de la experiencia cotidiana. 
REVISTA DE LA ESCUELA DE CIENCIAS DE LA EDUCACIÓN, AÑO 17, NRO. 16, VOL. 2, JULIO A DICIEMBRE DE 2021. PÁGINAS 13-25. ISSN 2362-3349 (EN LÍNEA). POLIITICAS DE INCLUSIÓN EDUCATIVA DE ADOLESCENTES, JÓVENES Y ADULTOS EN LA PROVINCIA DE BUENOS AIRES: TENSIONES EN TORNO A LA SEGMENTACIÓN SOCIO EDUCATIVA Y EL ACCESO AL CONOCIMIENTO. JORGELINA SILVIA SASSERA. NATALIA HERGER

Desde un enfoque social realista, el autor reflexiona sobre las posturas que se enfocan principalmente en los educandos y su experiencia, pues los estudiantes no pueden construir su propio aprendizaje y el rol de los docentes no debería reducirse al de facilitadores en vez de fuentes de conocimiento especializado (Young \& Muller, 2010). Estos argumentos advierten sobre las propuestas educativas que borran u ocultan las fronteras entre los distintos tipos de conocimiento y los lugares en que estos se adquieren, y en particular entre el conocimiento codificado que enseña la educación formal y el conocimiento experiencial.

La reflexión en torno al acceso al conocimiento radica en que "es tanto a) un asunto epistemológico que define el derecho de los estudiantes al acceso al sistema educativo y a los campos de especialización, como b) una cuestión de justicia social sobre el derecho al conocimiento de todos los estudiantes" (Riquelme; Young \& Muller, 2016). Así, la noción de curriculum como instancia para transmitir conocimiento es importante para interpretar las estrategias que pretenden garantizar el derecho a la educación en sentido complejo, es decir, no solo a través de ampliar el ingreso al sistema educativo sino también con estrategias para la apropiación de conocimiento para todos y no solo para algunos pocos.

Otros autores plantean que "la educación es un proceso social en el que el 'cuánto' no se puede separar del 'qué'. Existe un nexo ineludible entre distribución y contenido" (Connell, 1997, p. 27). La justicia distributiva en educación debería asentarse en el logro de la justicia curricular. Esto implica un currículum común para todos los ciudadanos y construido teniendo en cuenta al menos tres principios: la expresión clara de los intereses de los grupos menos favorecidos, la participación de todos los sectores sociales y la producción histórica de la igualdad. De acuerdo con Connell, una "desigual distribución de la educación entre clases sociales" está asociada a la existencia de diferentes currículum" (Connell, 1997, p. 28). Este autor parece coincidir con Young, en la importancia de acceso igualitario al conocimiento socialmente valorado y las disciplinas como recursos para superar las desigualdades educativas.

En Argentina, así como en otros países de América Latina, la existencia de importantes sectores de la población que deben terminar la primaria y la secundaria implica reconocer vulneración del derecho a la educación y la persistencia de una "deuda social educativa" con los niños, adolescentes, jóvenes y adultos excluidos del sistema educativo (Riquelme, 2013; Riquelme \& Kodric, 2013). Esta población está en una posición de desventaja social pues no ha podido apropiarse de los conocimientos, aptitudes y destrezas necesarios para participar en la vida ciudadana.

Los discursos de la obligatoriedad, la gratuidad y su formalidad tienen limitaciones pues no garantizan las condiciones de la permanencia en los sistemas educativos y que mantienen su cariz tradicionalmente excluyente. De acuerdo con Gentili (2009) los avances en la garantía del derecho a la educación en Latinoamérica han configurado un proceso de "exclusión incluyente" caracterizada por una universalización de oportunidades y expansión del acceso a la escuela en contextos de déficit de condiciones adecuadas para el aprendizaje con dinámicas de inclusión insuficientes para revertir los procesos de marginación y negación de derechos. En estos contextos de segmentación socioeducativa no se ha logrado democratizar el acceso al conocimiento socialmente disponible para todos, que es monopolizado por grupos o sectores que disponen de los recursos y del poder para acumularlo.

La segmentación socio-educativa afectaría las posibilidades de una distribución y apropiación universal de los contenidos educativos por parte de los grupos sociales tanto por las diferencias en el acceso y la permanencia como por las condiciones desiguales en que se desarrolla la enseñanza y el aprendizaje.

De acuerdo con Terigi (2009) pueden reconocerse al menos tres situaciones que afectan el acceso igualitario al conocimiento: las formas de escolaridad de baja intensidad, el currículum centrado en aprendizajes elitistas o sectarios y los aprendizajes de baja relevancia que ocurren cuando los alumnos acceden a versiones devaluadas de los contenidos curriculares. Los estudiantes que experimentan estos tipos de escolaridad ingresarían al sistema educativo e incluso completarían los niveles obligatorios con la obtención de los títulos correspondientes, pero los conocimientos adquiridos limitarían las posibilidades de completar la educación superior y/o universitaria o construir otros proyectos de formación autónomos.

Estas cuestiones llevan a preguntarse por los alcances y limitaciones de las políticas de inclusión educativa dirigidas a jóvenes y adultos, en tanto no se trataría solo de contemplar la expansión del acceso y la finalización del nivel sino de incidir en las prácticas escolares y en el currículo para superar las desigualdades en la distribución del conocimiento.

\section{Las políticas de inclusión educativa de jóvenes y adultos en las últimas dos décadas: un acercamiento a las condiciones de acceso al conocimiento en el Plan FinEs en la provincia de Buenos Aires}

Este apartado plantea avances en la reflexión sobre la relación entre las políticas recientes de inclusión educativa de jóvenes y adultos en Argentina con los procesos de segmentación socioeducativa, considerando las características del surgimiento, implementación y propuesta pedagógica del Plan de Finalización de Estudios Primarios y Secundarios FinEs, y específicamente las características de su implementación en la Provincia de Buenos Aires. Este plan dirigido a adultos que no finalizaron su educación básica plantea trayectos individualizados de cursadas de materias por módulos breves en el marco de formas institucionales alternativas a las ofertas regulares de secundaria y secundaria de adultos.

En los 2000, luego de la crisis económica y social consecuencia de las políticas neoliberales de la década previa, en Argentina y otros países de América Latina se recupera el rol del Estado en el planteo 
REVISTA DE LA ESCUELA DE CIENCIAS DE LA EDUCACIÓN, AÑO 17, NRO. 16, VOL. 2, JULIO A DICIEMBRE DE 2021. PÁGINAS 13-25. ISSN 2362-3349 (EN LÍNEA). POLIITICAS DE INCLUSIÓN EDUCATIVA DE ADOLESCENTES, JÓVENES Y ADULTOS EN LA PROVINCIA DE BUENOS AIRES: TENSIONES EN TORNO A LA SEGMENTACIÓN SOCIO EDUCATIVA Y EL ACCESO AL CONOCIMIENTO. JORGELINA SILVIA SASSERA. NATALIA HERGER

de políticas públicas que favorezcan la ampliación de derechos y la inclusión social (Feldfeber \& Gluz; 2019). En esta década el discurso político educativo y las medidas implementadas remarca el derecho social a la educación y la inclusión de la población, especialmente en la educación secundaria en tanto nivel obligatorio.

Ello se expresa en la extensión de la obligatoriedad hasta el nivel secundario, reconocida por la Ley de Educación Nacional ( $\left.N^{\circ} 26.206 / 2006\right)$ que instala con fuerza la responsabilidad del Estado en garantizar la oferta de servicios educativos a todos aquellos que no han completado su escolaridad. La concreción del derecho a la educación generó grandes desafíos en un sistema educativo fragmentado en tantos sub-sistemas como provincias y que debía atravesar por una nueva "transición crítica" (Riquelme, 2004), que implicó cambios en la estructura de niveles y ciclos, en los planes de estudios y en los títulos y certificaciones.

Algunos autores señalan que a partir de este momento la inclusión educativa no es considerada sólo como una ampliación del acceso sino que también requiere replantearse las instituciones y las estrategias de 'acogida (Dussel; 2015). Otros trabajos sostienen que corresponde a una etapa de "transición del modelo compensatorio hacia un formato todavía indefinido, que busca una nueva síntesis entre lo universal y la heterogeneidad social" (Rivas, Veleda \& Mezzadra; 2011, p. 29). Estos autores plantean que las denominadas políticas "socioeducativas", rescatan la intención de lograr un abordaje social y pedagógico integral ante las desigualdades en las condiciones de aprendizaje de la población, pero manteniendo un diseño focalizado en las escuelas más vulnerables.

En este período se desarrollaron una diversidad de políticas y programas educativos dirigidos a sostener la escolaridad de niños, adolescentes y jóvenes, a la par que desde las políticas sociales se implementaron programas de transferencia condicionada de ingresos para los hogares y poblaciones en mayor desventaja socioeconómica que contenían contraprestaciones ligadas a la asistencia escolar y/o la formación laboral. Así, algunos investigadores sostienen que estos programas (AUH y luego el PROG.R.ES.AR) dan cuenta de un nuevo paradigma de "protección ampliada" desde la infancia hasta la juventud (Mazzola, 2014).

La educación secundaria obligatoria constituyó y todavía constituye un desafío al que se orientaron diversas acciones que buscaron garantizar el acceso, permanencia y finalización del nivel, y que se sustentaron en el Plan Nacional de Educación Obligatoria de 2009 y en los planes de años posteriores. Los acuerdos federales en el CFE dieron- y dan- lugar a algunas experiencias de modificaciones del formato escolar o de formas "innovadoras" de organización de la secundaria para superar los límites de la "escuela tradicional" y acoger a nuevos grupos de población anteriormente excluidos de este nivel.

El esfuerzo por escolarizar a los sectores excluidos del sistema educativo en el marco de la secundaria obligatoria enfrentaría múltiples obstáculos, ya que, como señalan algunos trabajos este nivel tiene una matriz selectiva que choca con los imperativos de universalización de la normativa vigente (Terigi, 2011), de allí la formulación de diversas iniciativas y experiencias que se proponen incluir, establecer puentes, acelerar tiempos y trayectorias y acompañar a los jóvenes y adultos que se encontraban por fuera del sistema.

Un aspecto de estas propuestas es la flexibilización, que aparece como una opción para posibilitar la inclusión socioeducativa de los jóvenes y adultos que quedan al margen del sistema educativo debido a la rigidez de sus normas, tradiciones, pautas de distribución del conocimiento, evaluación y acreditación o desconocimiento del régimen académico (Baquero et. al. 2009). En algunos estudios se plantea si estos cambios constituirían la expresión de un patrón institucional diferenciado para la inclusión de determinados grupos sociales (Riquelme, Herger \& Sassera, 2018a; Riquelme, 2004; Tiramonti, 2008).

\section{Las políticas de inclusión y los cambios políticos educativos. El caso de FinEs en la provincia de Buenos Aires}

El reconocimiento de la fragmentación del sistema educativo y del aumento de la desigualdad educativa derivadas de las políticas neoliberales, llevó a la discusión e instalación de la problemática del derecho a la educación desde finales de los '90 y después de la crisis del 2001, en que se empezaron a concretar en algunas medidas de política (Riquelme, Herger \& Sassera, 2018a y Riquelme \& Herger, 2009). Desde 2005, se inició una nueva transición crítica del sistema educativo y la educación secundaria a partir de la sanción de la Ley de Financiamiento Educativo N²6.075/2005; Ley de Educación Técnico-Profesional (26.058/2005) y la Ley de Educación Nacional (LEN) N²6.206/2006.

En este período la educación de jóvenes y adultos es revalorizada en la LEN, al establecerla como una de las modalidades educativas y dar el marco para la definición y orientaciones de acciones de promoción de la alfabetización, vuelta a la escuela y terminalidad de los niveles obligatorios. Tanto el Plan de Alfabetización "Encuentro" y especialmente el FinEs, expresaron una intención de adecuación a la diversidad y especificidad de situaciones educativas de los jóvenes y adultos, con criterios de disponibilidad, accesibilidad y distribución en el territorio de cada jurisdicción. También se acordaron marcos normativos y curriculares así como acciones para el fortalecimiento de la gestión y planificación de la modalidad.

FinEs constituyó desde 2008, la principal medida dirigida para que los jóvenes y adultos que no continuaron la secundaria, completen el nivel. Esta iniciativa nacional fue traducida en cada provincia con variaciones en la implementación y no se dispone aún de información sobre la cobertura alcanzada en los 
REVISTA DE LA ESCUELA DE CIENCIAS DE LA EDUCACIÓN, AÑO 17, NRO. 16, VOL. 2, JULIO A DICIEMBRE DE 2021. PÁGINAS 13-25. ISSN 2362-3349 (EN LÍNEA). POLITICAS DE INCLUSIÓN EDUCATIVA DE ADOLESCENTES, JÓVENES Y ADULTOS EN LA PROVINCIA DE BUENOS AIRES: TENSIONES EN TORNO A LA SEGMENTACIÓN SOCIO EDUCATIVA Y EL ACCESO AL CONOCIMIENTO. JORGELINA SILVIA SASSERA. NATALIA HERGER

distintos territorios y en el total del país ${ }^{3}$. El Plan comenzó como un programa a término o transitorio, pero la alta demanda hizo que se extendiera en el tiempo sin una adecuada planificación o previsión y sin contar con los recursos necesarios; además son disputadas en su legitimidad por algunos actores del sistema educativo.

Un aspecto significativo del plan es que sus sedes no necesariamente son instituciones educativas, sino que se ha desarrollado en otras organizaciones de la sociedad civil en convenio con el Ministerio de Educación. Esta particularidad ubicó al FinES como una propuesta paralela al sistema regular de educación de adultos, a veces en competencia y otras veces en cooperación desde los márgenes del sistema.

El cambio de gobierno en 2015 significó la asunción de un partido con una agenda política, económica y social neoliberal, que planteó fuerte críticas a la educación pública y puso en jaque la continuidad de las políticas de inclusión educativa del período anterior, generando el rechazo de gran parte de la comunidad educativa. En este período se redujo el financiamiento educativo, y el derecho a la educación se desplazó hacia una perspectiva individual y ligada a la noción de meritocracia. A la par, muchos programas previos continuaron vigentes aunque con alcances o coberturas más restringidas y otros, como el Progresar, se convirtieron en becas asignadas de acuerdo al rendimiento de los estudiantes.

El Plan FinEs fue prorrogado por el Ministerio de Educación y Deportes de la Nación para el período 2016-2019 con tres líneas de acción - deudores materias, trayecto primaria y trayecto secundaria para mayores de 18 años- y con algunos cambios con respecto a los aspectos regulatorios de la implementación del Plan en las jurisdicciones. El Ministerio nacional implementaría un sitio web para la preinscripción para los interesados y de un sistema de información para la mejora en la planificación, ejecución, seguimiento y evaluación de las dimensiones pedagógica, institucional y administrativa en las jurisdicciones del país ${ }^{4}$. Se buscó modificar aspectos de regulación en las sedes y en instancias de jurisdiccionales al establecer figuras de "responsables", y proveer de asistencia técnica y financiamiento para docentes, tutores y secretarios. En este sentido, se podría entrever que el gobierno nacional asumiría un rol evaluador y de mayor seguimiento de la implementación del plan en cada jurisdicción.

En la provincia de Buenos Aires, el FinEs tuvo una traducción particular, primero dentro del Plan Provincial y luego a través del llamado Fines2, dirigido a trabajadores incluidos en programas sociales ${ }^{5} y$ en entidades gremiales. La transformación del programa a lo largo del tiempo, tanto en los componentes que lo integran y en las distintas dependencias y denominaciones con el que se lo conocen, generaron confusiones y dificultades entre los interesados en concurrir (Toscano y Serial, 2012). Las sucesivas traducciones y re-contextualizaciones ocurridas en los distintos espacios locales, también contribuyeron a la desorganización en la implementación de la política, a las confusiones en la forma de nombrarlo, a su gestión cotidiana y a cierta deslegitimación del Plan proveniente desde algunos actores del mismo sistema educativo (Herger \& Sassera, 2016).

A partir de 2016, la nueva gestión de la provincia de Buenos Aires, introduce una serie de cambios en la operatoria del Plan, que si bien escapan a los objetivos de este articulo, pueden mencionarse brevemente: el programa pasó a depender exclusivamente de la Dirección de Educación de Jóvenes y Adultos de la DGCyE, área desde la que se encaró una "reorganización" que supuso que las sedes de FinEs dependieran de los Centros de Educación de Nivel Secundario, de las escuelas primarias de adultos y las comisiones que funcionaban en organizaciones sociales, sindicales etc. pasaran a ser "aulas externas". Otros cambios fueron la división del plan en tres áreas (territorial, pedagógica y administrativa), la definición de coordinadores regionales, administrativos y pedagógicos, la selección de los docentes a través del listado oficial y el aumento de la carga horaria (de 13 a 18 hs. cátedra) ${ }^{6}$.

Existen estudios que señalan la importancia de FinEs para la finalización de la educación secundaria, las posibilidades que brindó-y brinda- para la terminalidad educativa y la significación que tuvo y tiene para los sujetos que transitaron por él (Míguez, 2018; Burgos, 2016, Finnegan \& Brunetto, 2014). Los trabajos que abordan el alcance y cobertura del plan, la dimensión pedagógica, curricular y del desarrollo de los aprendizajes son escasos, y resulta necesario explorar algunas de esas cuestiones.

\footnotetext{
${ }^{3}$ El anuario estadístico educativo de 2018 es el primero que incluye indicadores sobre estudiantes y trayectoria en el Plan para algunas provincias.

${ }^{4}$ Res. 178/2016.

${ }^{5}$ Especialmente a los trabajadores de las cooperativas del Programa Ingreso Social con trabajo, Argentina Trabajo.

${ }^{6}$ Resolución DGCyE 713/17.
} 
REVISTA DE LA ESCUELA DE CIENCIAS DE LA EDUCACIÓN, AÑO 17, NRO. 16, VOL. 2, JULIO A DICIEMBRE DE 2021. PÁGINAS 13-25. ISSN 2362-3349 (EN LÍNEA). POLITICAS DE INCLUSIÓN EDUCATIVA DE ADOLESCENTES, JÓVENES Y ADULTOS EN LA PROVINCIA DE BUENOS AIRES: TENSIONES EN TORNO A LA SEGMENTACIÓN SOCIO EDUCATIVA Y EL ACCESO AL CONOCIMIENTO. JORGELINA SILVIA SASSERA. NATALIA HERGER

Cuadro 2. Unidades educativas y alumnos de la educación de jóvenes y adultos Provincia de Buenos Aires. Absolutos y porcentajes. 2017 a 2019.

\begin{tabular}{|l|c|c|c|c|c|c|}
\hline \multirow{2}{*}{$\begin{array}{l}\text { Educación de } \\
\text { Adultos }\end{array}$} & \multicolumn{2}{|c|}{$\mathbf{2 0 1 7}$} & \multicolumn{2}{c|}{$\mathbf{2 0 1 8}$} & \multicolumn{2}{c|}{$\mathbf{2 0 1 9}$} \\
\cline { 2 - 7 } & $\begin{array}{c}\text { Unidades } \\
\text { Educativas }\end{array}$ & Alumnos & $\begin{array}{c}\text { Unidades } \\
\text { Educativas }\end{array}$ & Alumnos & $\begin{array}{c}\text { Unidades } \\
\text { Educativas }\end{array}$ & Alumnos \\
\hline Total & $\mathbf{2 . 0 6 8}$ & $\mathbf{4 4 3 . 5 7 4}$ & $\mathbf{1 . 9 3 0}$ & $\mathbf{4 5 8 . 0 9 4}$ & $\mathbf{2 . 0 0 3}$ & $\mathbf{5 1 0 . 9 3 6}$ \\
\hline Nivel Primario & 589 & 51.392 & 593 & 72.668 & 577 & 78.917 \\
\hline $\begin{array}{l}\text { Nivel } \\
\text { Secundario }\end{array}$ & 893 & 163.317 & 820 & 126.939 & 844 & 129.732 \\
\hline $\begin{array}{l}\text { Plan Fines } \\
\text { (Trayectos y } \\
\text { Deudores) }\end{array}$ & 123 & 43.203 & 57 & 68.195 & 126 & 95.354 \\
\hline $\begin{array}{l}\text { Formación } \\
\text { Profesional }\end{array}$ & 463 & 185.662 & 460 & 190.292 & 456 & 206.933 \\
\hline
\end{tabular}

Fuente: elaboración propia sobre la base de DGCyE, Relevamiento Anual, 17, 2018 y 2019

Los datos disponibles para el período 2017-2019, permiten observar un aumento de la matrícula del Plan Fines y una disminución de la correspondiente a la educación de jóvenes y adultos de nivel secundario. Cabe destacar, que este crecimiento de la matrícula del Plan, no fue acompañado con el aumento de las sedes, lo cual genera el interrogante sobre las condiciones de atención de los estudiantes. El alcance de estos datos debería contrastarse con la demanda social, es decir, población que no completó la educación obligatoria, y que en la provincia de Buenos Aires alcanzaba a cerca de un millón (949.968) de jóvenes de 18 a 29 años ${ }^{7}$.

Cuadro 3. Unidades educativas, alumnos y secciones en el Gran buenos Aires e interior de la provincia. Absolutos. 2019.

\begin{tabular}{|l|c|c|c|}
\hline $\begin{array}{l}\text { Plan Fines (Trayectos y } \\
\text { Deudores) }\end{array}$ & $\begin{array}{c}\text { Unidades } \\
\text { Educativas }\end{array}$ & Alumnos & Secciones \\
\hline Total provincia & $\mathbf{9 8}$ & $\mathbf{9 5 . 3 5 4}$ & $\mathbf{2 . 2 5 0}$ \\
\hline Gran Buenos Aires & 49 & 50.207 & 1.147 \\
\hline Interior Buenos Aires & 77 & 45.147 & 1.103 \\
\hline
\end{tabular}

Fuente: elaboración propia sobre la base de DGCyE, Relevamiento Anual 2019

La distribución de la matrícula y de las unidades educativas en las que funciona FinEs, da cuenta que en el Gran Buenos Aires existe una menor cantidad de sedes pero atienden a más de la mitad del total de los estudiantes. Ello en parte refiere a la mayor concentración de jóvenes y adultos que no completaron el primario o el secundario en los distritos del GBA. Sin dudas, una mejor consideración de la cobertura lograda por el FinES y en relación a la educación de jóvenes y adultos regular requeriría contar con información de etapas previas de su implementación en la provincia y en el resto del país.

\section{La organización del plan de estudios en FinEs y las innovaciones pedagógicas}

El Plan FinEs se puede considerar una variación de la forma escolar, con cambios o innovaciones respecto a la organización de los estudiantes, la duración de las propuestas formativas y el reconocimiento de las trayectorias entre otras. La propuesta pedagógica se centra en el desarrollo de tutorías, diseñadas para la atención de jóvenes y adultos con trayectorias diversas. Según la disciplina, el tutor/docente acompaña grupal e individualmente a los estudiantes para "problematizar 'ejes o núcleos temáticos definidos por la currícula'; y guiar a cada uno de los cursantes en un proceso que le permita construir puentes entre los 'saberes y conocimientos de la vida cotidiana y el mundo laboral' y los 'saberes escolares" (Diez, 2012, p. 89).

Algunos trabajos señalan que es preciso puntualizar qué aspectos flexibilizan estos planes y programas, pues se tiende a flexibilizar el modelo organizacional sin el acompañamiento de cambios pedagógicos o curriculares (Terigi, 2011; Saccone, 2016). Una investigación sobre el Plan Provincial en el que se observaron tutorías y el desarrollo de actividades (Diez, 2012), advierte que no se habrían realizado los cambios esperados en el modelo pedagógico, sino que los docentes, probablemente debido a su formación y experiencia, siguen replicando las prácticas educativas propias del secundario común pero en una situación de mayor heterogeneidad del alumnado. Otros autores (Burgos, 2016) señalan que las innovaciones del Plan corresponden a la organización del tiempo escolar, en especial la carga horaria y la cursada semipresencial respecto a los bachilleratos de adultos.

${ }^{7}$ Estimación realizada para los partidos del Gran Buenos Aires y aglomerados del interior de la provincia sobre la base de la Encuesta Permanente de Hogares, $1^{\circ}$ trimestre de 2018. 
REVISTA DE LA ESCUELA DE CIENCIAS DE LA EDUCACIÓN, AÑO 17, NRO. 16, VOL. 2, JULIO A DICIEMBRE DE 2021. PÁGINAS 13-25. ISSN 2362-3349 (EN LÍNEA). POLIITICAS DE INCLUSIÓN EDUCATIVA DE ADOLESCENTES, JÓVENES Y ADULTOS EN LA PROVINCIA DE BUENOS AIRES: TENSIONES EN TORNO A LA SEGMENTACIÓN SOCIO EDUCATIVA Y EL ACCESO AL CONOCIMIENTO. JORGELINA SILVIA SASSERA. NATALIA HERGER

Uno de los puntos del régimen escolar o del modelo organizacional que flexibiliza FinEs es la carga horaria y los momentos de cursada respecto del régimen de los bachilleratos de adultos de la provincia. El plan de estudios de los bachilleratos bonaerenses se fundamenta en que "una escolarización excesiva provoca deserción y no tiene en cuenta el marco referencial del adulto" (Resolución de la DGCyE $\left.n^{\circ} 6321 / 95\right)$. El plan de estudios está estructurado en materias de formación general o fundamentación, orientadas y de formación especializada, implica una cursada de tres años de duración y prevé una carga horaria de 26 horas semanales, versus la secundaria común que tiene una duración de 5 o 6 años según la provincia.

La flexibilización curricular de FinEs Trayecto Secundario (conocido como Fines2) en provincia de Buenos Aires hasta 2017 mantenía los tres años de cursada - 6 cuatrimestres, 16 encuentros- y aunque se sostienen las mismas asignaturas con excepción de Química, debían dictarse en casi la mitad de horas previstas en el diseño curricular de los bachilleratos de educación secundaria de jóvenes y adultos. A partir de 2017, se producen cambios en la organización curricular del plan, por ejemplo se duplican la cantidad de horas para Matemáticas y Lengua y Literatura, es decir, materias básicas; y otras materias aumentaron una hora cátedra- por ejemplo Biología, Historia y Geografía, Educación Cívica, Física. La formación especializada que dependía de la oferta de la institución, fue reemplaza por materias pautadas y con mayor cantidad de horas que la anterior, tales como Economía social, Políticas Públicas y Derechos Humanos, Estado y políticas públicas, Diseño y desarrollo de proyectos, Estado y nuevos movimientos sociales. Otras modificaciones apuntan al aumento de la duración del plan de estudios (a 32 horas cátedra), a la exigencia de una mayor presencialidad de los estudiantes, que aumenta de dos a tres días; y se mantiene el requisito de asistir al $75 \%$ de las clases.

Antes de estos cambios, las propuestas pedagógicas para cada materia eran realizadas por los docentes tutores, seleccionados de acuerdo a su formación docente y trayectoria. Según una investigación, los criterios para la valoración de un proyecto pedagógico era la solidez teórica de su fundamentación y su factibilidad, los modos de evaluación seleccionados, la bibliografía elegida, la estructuración de los contenidos y la consideración de las características del alumnado (Diez, 2012). Los cambios dispuestos desde 2017 indican los requisitos específicos para la presentación de los proyectos pedagógicos y un detalle de los apartados que deben incluir.

La flexibilización alcanza también a las pautas de evaluación. Si bien formalmente los alumnos deben entregar dos trabajos prácticos obligatorios y aprobar un trabajo de integración de $\operatorname{conocimientos}^{8}$; los docentes realizan adaptaciones de las modalidades de evaluación, pudiendo consistir en trabajos presenciales, individuales, grupales en clase o domiciliarios.

A pesar de la ampliación de las horas de cursada existen dificultades para los docentes en términos del desarrollo y jerarquización de contenidos. En un estudio se señala que los profesores expresaron dificultades al tener que desarrollar los contenidos de los programas pues "los planes de estudio han sido una traslación de los lineamientos curriculares de la Educación Permanente de Jóvenes y Adultos en la enseñanza secundaria para adultos condensando casi los mismos contenidos en menos horas de clase" (Secretaría de Evaluación de la Calidad Educativa, 2018, p. 69). Estas citas reflejan las tensiones entre el "cuánto" y el "qué" enseñar (Connell, 1997), que no alcanzan a resolverse solo con una mayor la cantidad de horas de dictado, y parecen referir a la necesidad de una selección de contenidos relevantes en términos de la adecuación a la propuesta pedagógica del Plan.

La relación de los estudiantes con los contenidos en este ámbito es también compleja. El mismo estudio señala que los estudiantes de FinEs presentan dificultades en cuanto a la escritura, la incorporación de nuevos aprendizajes, la expresión y comunicación debido, en muchos casos, al alejamiento durante largos períodos del sistema educativo. En algunos casos rechazan contenidos y los docentes deben "convencer" a los estudiantes sobre el valor y utilidad de los temas de las materias (Secretaría de Evaluación de la Calidad Educativa, 2018). De acuerdo con estos estudios, algunos estudiantes parecen cuestionar el valor social de los contenidos que se les proponen y requerir una mayor contextualización de los mismos en relación con sus condiciones de vida actuales y proyectos futuros.

Los cambios a partir de 2017 parecen intentar una respuesta a los señalamientos respecto a la necesidad de mayor cantidad de horas de dictado de las materias y de una mayor presencialidad de los estudiantes, lo cual podría contribuir a dar una mayor solidez a la experiencia educativa en el marco de una relación de enseñanza y aprendizaje más extensa y continua entre docente y estudiantes. La reciente aplicación de estas modificaciones no hace posible contar aún con investigaciones acerca de su posible incidencia respecto a las formas en que se produce la selección de los contenidos para la enseñanza y la apropiación por parte de los estudiantes.

\section{Tensiones entre la fragilidad institucional, el acceso al conocimiento y el cumplimiento al derecho a la educación}

La población joven y adulta de la provincia que no finalizó la educación secundaria, se enfrenta con una diversidad de opciones en la oferta educativa vigente, que pueden interpretarse como una fragmentación y diferenciación de la misma. En el caso de los adolescentes, se abrieron instancias de terminalidad y reingreso a la educación secundaria mediante bachilleratos juveniles y opciones de

\footnotetext{
${ }^{8}$ Res. DGCyE 713/17.
} 
REVISTA DE LA ESCUELA DE CIENCIAS DE LA EDUCACIÓN, AÑO 17, NRO. 16, VOL. 2, JULIO A DICIEMBRE DE 2021. PÁGINAS 13-25. ISSN 2362-3349 (EN LÍNEA). POLITICAS DE INCLUSIÓN EDUCATIVA DE ADOLESCENTES, JÓVENES Y ADULTOS EN LA PROVINCIA DE BUENOS AIRES: TENSIONES EN TORNO A LA SEGMENTACIÓN SOCIO EDUCATIVA Y EL ACCESO AL CONOCIMIENTO. JORGELINA SILVIA SASSERA. NATALIA HERGER

aceleración a través de propuestas educativas no graduadas para la educación secundaria común y la educación secundaria técnica. Los adultos mayores de 18 años disponen de los CENS, que ofrecen una mayor institucionalidad para la finalización de los estudios, junto a programas con mayor trayectoria como FinEs y otros programas de reciente creación ${ }^{9}$ de los que aún no hay análisis suficientes (Herger \& Herger, 2019).

La implementación de FinEs en Buenos Aires dio lugar a diversas investigaciones (Toscano \& Serial, 2012; Diez, 2012; Krichesky et. al. 2013; Finnegan \& Brunetto, 2015; Burgos, 2016; Gruszka \& Abritta, 2018; Míguez, 2018; Riquelme, Herger \& Sassera, 2018a). Algunos trabajos dan cuenta de la significación de las propuestas de terminalidad educativa en términos de las oportunidades que brindan a los jóvenes y adultos para la finalización de sus estudios y el cumplimiento del derecho a la educación, pero en el marco de un sistema educativo segmentado (Herger \& Sassera, 2016; Riquelme, Herger \& Sassera, 2018a).

Un interrogante es si FinEs constituye una opción diferenciada que permite incluirlo en un trayecto o circuito educativo de baja intensidad y fragilidad institucional. Así, este programa podría expresar la contradicción entre la intención política de homogeneización de la educación secundaria, mientras que a la par se abren propuestas específicas que flexibilizan el formato de la escuela con el objetivo de incluir a grupos en situación de vulnerabilidad. Además se formulan roles docentes con distintas pautas de contratación y tareas pedagógicas, contenidos adaptados a estos contextos y poblaciones, y articulaciones con otros niveles y modalidades de la educación.

Algunos estudios señalan que la reducida cantidad de días y horas de cada materia, así como la flexibilización de la cursada, despertó a lo largo de los años numerosas críticas en torno a qué aprendizajes se promueven y se apropian los jóvenes y los adultos que acceden a FinEs: los actores del propio sistema educativo lo señalan como una instancia que deterioraría la calidad de los conocimientos enseñados y una competencia para la oferta regular de educación de adultos pues sería "más fácil" (Krichesky, 2013; Toscano \& Diez, 2012)

Los docentes responsables de implementar el plan, han planteado también la preocupación acerca las posibilidades de acceso al conocimiento de la población en un marco de justicia curricular (Connell, 1997) que debiera ser garantizado mediante políticas adecuadamente planificadas y financiadas:

"Esto es una dificultad para la gestión del programa porque su llegada a secundaria que se da en el marco de una estructura que no lo puede sostener y de directores que se ocupan del financiamiento, de la organización de los recursos y demás, es un escenario poco probable para un programa o para FinEs" (Inspector, Berazategui, 2015)

Estas tensiones contrastan con el reconocimiento del programa por parte de los docentes, referentes, coordinadores y estudiantes en términos de las posibilidades brindadas para la finalización de los estudios secundarios:

"-Fines vino a desenterrar el analfabetismo, el impacto que generó fue muy masivo y aparte en un primer momento no era muy bien visto y después...- fue ganando visibilidad. Me parece que el impacto fue porque se acercó al lugar donde estaba la necesidad.

- fue a la puerta del ciudadano, ahí está la diferencia con el sistema formal” (Inspectores, Florencio Varela, $2015)^{12}$

Míguez (2018) reconstruye a partir del relato de estudiantes aspectos positivos del Plan, que contiene y motoriza el objetivo de finalizar los estudios: la puesta en valor de los saberes previos, el aprendizaje colectivo y el vínculo construido entre estudiantes y profesores a través del encuentro y la conformación de grupos. Así, diferentes estudios resaltan que FinEs, es una experiencia significativa para reconocer aquellos aspectos de los formatos escolares flexibilizados que fomentan el aprendizaje y que logran sostener la escolaridad de los jóvenes y adultos.

\section{A modo de cierre}

El derecho a la educación fue una orientación en la últimas décadas de la política educativa de Argentina y se expresó en la sanción de Ley de Educación Nacional, la extensión de obligatoriedad escolar hasta nivel secundario, el reconocimiento de la educación permanente y en la implementación de programas dirigidos a favorecer el acceso, permanencia y la vuelta al sistema educativo. Estas medidas plantean tensiones y contradicciones pues se asentaron en un sistema segmentado generador de nuevas desigualdades vinculadas a la diversificación de la oferta educativa y a las condiciones diferenciales para la apropiación de conocimientos.

Los estudios realizados dan cuenta que las políticas que apuntan a la atención educativa de las poblaciones excluidas, crean formas institucionales alternativas que pueden constituir un circuito caracterizado por la fragilidad de los recursos materiales, condiciones de trabajo de los docentes y

\footnotetext{
${ }^{9}$ Los programas de educación de jóvenes y adultos vigentes en 2019 en la provincia eran Programa Familias a Estudiar, Programa Adultos 3.0, Programa Secundaria con Oficios, Programa Terminalidad Secundaria para Formación Profesional y Plataforma Virtual con Tutorías.

${ }^{10}$ Las citas son tomadas de entrevistas realizadas a inspectores de educación secundaria y de educación de jóvenes y adultos, coordinadores de FinEs y de asistentes administrativos realizadas en el marco del proyecto a citar.
} 
REVISTA DE LA ESCUELA DE CIENCIAS DE LA EDUCACIÓN, AÑO 17, NRO. 16, VOL. 2, JULIO A DICIEMBRE DE 2021. PÁGINAS 13-25. ISSN 2362-3349 (EN LÍNEA). POLITICAS DE INCLUSIÓN EDUCATIVA DE ADOLESCENTES, JÓVENES Y ADULTOS EN LA PROVINCIA DE BUENOS AIRES: TENSIONES EN TORNO A LA SEGMENTACIÓN SOCIO EDUCATIVA Y EL ACCESO AL CONOCIMIENTO. JORGELINA SILVIA SASSERA. NATALIA HERGER

capacidades de gestión institucional que redundan en una "baja intensidad institucional". Las condiciones materiales, políticas y pedagógicas diferencian el acceso y permanencia de la población en los distintos circuitos educativos con diferentes posibilidades de articulación y pasaje entre sí que habilitan- o no- a una socialización compartida.

Si bien los programas de inclusión implementados en las últimas décadas han sido objeto de varios estudios, son escasos los trabajos que dan cuenta de los aspectos pedagógicos y menos aún los referidos al curriculum y de la selección de los contenidos en las propuestas presentadas por los docentes tutores. Sin dudas, ello requiere de estudios específicos que incluyan dimensiones tales como:

- los contenidos de cada materia y cómo se realiza la selección y adaptación en las propuesta pedagógicas del plan;

- qué actividades y estrategias desarrollan los docentes;

- las propuestas de evaluación y los logros de los estudiantes.

El programa analizado, se asienta en la flexibilización de las condiciones para la entrada, permanencia y finalización de la educación secundaria, especialmente la reducción de los tiempos de cursada en relación con la extensión de la educación de jóvenes y adultos. Según los antecedentes bibliográficos consultados, los docentes y otros actores intervinientes marcan como aspectos pendientes la definición de pautas y guías para la selección y organización de los contenidos, las actividades de enseñanza y evaluación y la capacitación de los tutores. Los cambios en la organización e implementación del Plan en la Provincia de Buenos Aires no parecen atender o apuntar a estas cuestiones, que quedan a criterio de cada docente. El incremento en la cantidad de horas para algunas materias es uno de los elementos a considerar respecto a generar condiciones de acceso a los conocimientos para los jóvenes y adultos que transitan por estas ofertas.

El diseño de propuestas educativas más flexibles que atiendan a las características de los jóvenes y adultos es sin dudas una condición fundamental para lograr la vuelta a la escuela así como la concreción del derecho a la educación. Sin embargo, es también importante garantizar las posibilidades de apropiación de saberes socialmente relevantes o valorados, equivalentes a los del resto del sistema.

Ello pone un alerta sobre la posible conformación de circuitos de baja intensidad y/o en los que se transmiten y aprenden contenidos curriculares diferenciales para los grupos sociales en mayor desventaja socioeconómica, y que no parecen garantizar el acceso al conocimiento poderoso a toda la población en tanto condición para el logro de la justicia social y equidad distributiva. Los estudios citados en este artículo así como las entrevistas realizadas en nuestra investigación ponen en evidencia las tensiones alrededor de estos programas en tanto que los actores educativos - los docentes y los estudiantes y los padres - y así como la sociedad en general perciben que constituyen una oportunidad de acceder y completar la educación obligatoria para jóvenes y adultos excluidos del sistema educativo, pero también advierten la devaluación de los contenidos que se enseñan y aprenden en los distintos circuitos y que afectan sus posibilidades de continuar estudios y de acceder al mercado de trabajo (Kessler, 2002; Dussel, 2007).

La idea de distribución ilusoria de la educación, da cuenta de la no concreción de la meta de acceso y permanencia de toda la población en el sistema educativo hasta completar al menos los niveles obligatorios. Esta meta no ha sido lograda aún ni en Argentina ni en otros países de la región latinoamericana, pese a su reconocimiento formal en las leyes desde fines del siglo XIX (Riquelme, Herger \& Sassera, 2018a). El derecho a la educación entendido como el acceso a todos los niveles educativos a lo largo de la vida y con contenidos socialmente relevantes (generales y científico tecnológicos) sigue siendo un desafío para la política educativa en nuestro país.

\section{Referencias Bibliográficas}

Braslavsky, C. (1985). La discriminación educativa en Argentina. Buenos Aires: FLACSO-Grupo Editor Latinoamericano. Braslavsky, C. \& Filmus D. (1987). Respuestas a la crisis educativa. Buenos Aires: Cántaro/FLACSO.

Baquero, R. et. al. (2009). Variaciones del régimen académico en escuelas medias con población vulnerable. Un estudio de casos en el Área Metropolitana de Buenos Aires. REICE Revista Iberoamericana sobre Calidad, Eficacia y Cambio en Educación, 4, 292- 319

Burgos, A. (2016). El Plan FinEs: un análisis de los aportes y límites en la ampliación del derecho a la educación en las políticas sociales. IX Jornadas de Sociología de la UNLP.

Connell, R. W. (1997). Escuelas y Justicia Social. Madrid: Ediciones Morata.

Diez, M. L. (2012). El plan en las escuelas. Toscano, A. G. (coord). Adolescentes y secundaria obligatoria Finalización de Estudios y Vuelta a la Escuela. Conurbano (Argentina). Buenos Aires: UNICEF.

Dussel, I. et. al. (2007). Más allá de la crisis. Visión de alumnos y profesores de la escuela secundaria argentina. Buenos Aires: Fundación Santillana.

Dussel, I. (2015). El rol de las organizaciones sociales en las políticas de inclusión socioeducativa. En: Faur, L. Repensar la inclusión social: políticas públicas y sociedad civil en la Argentina, 19991-2016.Buenos Aires: Capital Intelectual.

Feldfeber, M. \& Guz, N. (2019). Las políticas educativas a partir del cambio de siglo: Alcances y límites en la ampliación del derecho a la educación en la Argentina. Revista Estado y Políticas Públicas. 13.,19-38

Finnegan, F. \& Brunetto, C. (2014). "Acá hay un trabajo político": Actores y relaciones en la producción del Plan FinEs 2 Secundaria en la Provincia de Buenos Aires. VIII Jornadas de Sociología de la UNLP.

Filmus, D. (2001). Cada vez más necesaria, cada vez más insuficiente. Escuela media y mercado de trabajo en épocas de globalización. Buenos Aires: Santillana. 
REVISTA DE LA ESCUELA DE CIENCIAS DE LA EDUCACIÓN, AÑO 17, NRO. 16, VOL. 2, JULIO A DICIEMBRE DE 2021. PÁGINAS 13-25. ISSN 2362-3349 (EN LÍNEA). POLIITICAS DE INCLUSIÓN EDUCATIVA DE ADOLESCENTES, JÓVENES Y ADULTOS EN LA PROVINCIA DE BUENOS AIRES: TENSIONES EN TORNO A LA SEGMENTACIÓN SOCIO EDUCATIVA Y EL ACCESO AL CONOCIMIENTO. JORGELINA SILVIA SASSERA. NATALIA HERGER

Gallart, M. A. (2006). La construcción social de la escuela media. Una aproximación institucional. Buenos Aires: Editorial Stella y La Crujía Ediciones.

Gentili, P. (2009). Marcha y contramarchas. El derecho a la educación y las dinámicas de exclusión incluyente en América Latina (a sesenta años de la Declaración Universal de la Derecho Humanos). Revista lberoamericana de Educación, 49, 19-57.

Gruszka, M \& Abritta, A. (2018). La implementación del Plan FinEs: un análisis desde el trabajo docente. Revista Question, 1(59). pp. 1-18.

Herger, N. \& Sassera, J. (2016). Las políticas de atención del derecho a la educación para jóvenes y adultos y su traducción en espacios locales: alcances, limitaciones y tensiones, Dossier "Políticas educativas en América Latina en el siglo XXI. Balance y perspectivas". Revista del IICE, (40), 27-48. HYPERLINK "https://doi.org/10.34096/riice.n40.4131" https://doi.org/10.34096/riice.n40.4131

Herger, N. \& Sassera, J. (2019). Políticas recientes de educación y formación para el trabajo en la provincia de Buenos Aires. Continuidades, rupturas y escenarios complejos 2015-2018". 14 Congreso Nacional de Estudios del Trabajo, Asociación Argentina de Especialistas en Estudios del Trabajo, Buenos Aires, 7 al 9 de agosto de 2019 https://aset.org.ar/2019/contenido.htm

Kessler, G. (2002). La experiencia escolar fragmentada. Buenos Aires: IIPE-UNESCO.

Krichesky, M. (2013). La obligatoriedad de la educación secundaria en Buenos Aires. Perspectivas de la gestión y los profesores de la secundaria básica. Gonnet: UNIPE.

Llach. J. \& Schumacher, F. (2006). Segregación social en la educación primaria argentina. Llach, JJ. El desafío de la equidad educativa. Diagnóstico y propuestas. Buenos Aires: Granica, 75-112

Mazzola, R. (2014). PROG.R.ES.AR. Juventudes, bienes públicos y justicia distributiva. Revista Estado y Políticas Públicas, 2, 91-113.

Miguez, M. A. (2018). Experiencias de formación en el Plan Fines 2: un abordaje pedagógico desde la perspectiva (auto) biográfica y narrativa. En: Riquelme, G. C.; N. Herger y J. Sassera. Deuda social educativa con jóvenes y adultos. Entre el derecho a la educación, los discursos de las políticas y las contradicciones de la inclusión y la exclusión, Buenos Aires: Editorial de la Facultad de Filosofía y Letras, UBA.

Riquelme, G. C. (1985). Readaptación profesional y ocupacional de los trabajadores en contextos de crisis". Revista Argentina de Educación, 17-46.

Riquelme, G. C. (1989). Educación y trabajo en zonas desfavorables: reconceptualización y alternativas en la perspectiva de las políticas sociales. Buenos Aires: Ministerio de Educación y Justicia. OEA. PEM. Dirección General de Planificación Educativa.

Riquelme, G. C. (2004). La educación secundaria antes y después de la reforma: efectos distributivos del gasto público, Buenos Aires, FFyL/UBA- Miño y Dávila Editores.

Riquelme, G. C. (2013). La Deuda Social Educativa y el Derecho a la Educación: la importancia de los ejercicios de estimación de recursos para la planificación educativa. En: Riquelme, G. C. \& Kodric A. Deuda Social Educativa. Atención del derecho a la Educación. Ediciones Lumiere SA. Buenos Aires.

Riquelme, G. C. \& Herger, N. (2005). La doble exclusión educativa y laboral y los recursos individuales y sociales no apropiados por jóvenes y adultos en ámbitos locales: entre los diagnósticos tradicionales y los nuevos desafíos metodológicos. Serie de Cuadernos de Educación, Economía y Trabajo, 15. Buenos Aires, CONICET/PEETIICE (FFyL-UBA).

Riquelme, G. C. (in press). Review of "Young, Michael and Johan Muller, Curriculum and the specialization of knowledge. Studies in sociology of education", Mimeo

Riquelme, G. C. \& Herger, H. (2009). Educación y formación para el trabajo en la Argentina post-crisis: nuevas demandas y transición crítica en la educación secundaria. Novedades Educativas. 28. Recuperado de: https://peetiiceuba.files.wordpress.com/2016/06/2-riquelme-gc-y-herger-n-articulo-novedades-educativas.pdf

Riquelme, G. C. y Kodric, A. (2013). Deuda Social Educativa. Atención del derecho a la Educación. Ediciones Lumiere $\mathrm{SA}$. Buenos Aires.

Riquelme, G. C.; Herger, H. \& Sassera, J. (2018a). Deuda social educativa con jóvenes y adultos. Entre el derecho a la educación, los discursos de las políticas y las contradicciones de la inclusión y la exclusión. Buenos Aires: Editorial de la Facultad de Filosofía y Letras. Recuperado de: http://publicaciones.filo.uba.ar/deuda-socialeducativa-con-j\%C3\%B3venes-y-adultos.

Riquelme, G. C.; Herger, H. \& Sassera, J. (2018b). La atención educativa de la población en Argentina en las últimas décadas: entre la distribución ilusoria, la modernización eficientista y los discursos del derecho y la inclusión. Education Policy Analysis Archives, (158) 26. Recuperado de: https://doi.org/10.14507/epaa.26.3209

Rivas, A.; Veleda C. \& Mezzadra, F. (2011). La construcción de la justicia educativa. Criterios de redistribución y reconocimiento para la educación argentina, Buenos Aires, Argentina: CIPPECINICEF- Embajada de Finlandia.

Sassera, J. (2014). Educación y realidad social y productiva en ámbitos locales: el sentido de las experiencias pedagógicas de adolescentes y jóvenes, tesis de doctorado dirigida por la Dra. Graciela C. Riquelme. Doctorado de la Universidad de Buenos Aires, Área Ciencias de la Educación.

Saccone, M. (2016). La educación media en tiempos de transformaciones. Una mirada socioantropológica hacia México y Argentina. Buenos Aires: CLACSO.

Secretaría de Evaluación Educativa (2018). Investigación evaluativa del Plan Fines 2. Informe Final. Buenos Aires: Ministerio de Educación.

Subsecretaría de Educación (2008). Plan Provincial de Finalización de Estudios y Vuelta a la Escuela. Buenos Aires: DGCyE.

Tedesco, J. C. (comp.) (2005). ¿Cómo superar la desigualdad y la fragmentación del sistema educativo argentino? Buenos Aires: IIPE-UNESCO.

Terigi, F. (2011). Ante la propuesta de 'nuevos formatos': elucidación conceptual”. Quehacer educativo, 107, 15- 22.

Terigi, F. (2009). La inclusión educativa: viejas deudas y nuevos desafíos. Segmentación urbana y educación en América Latina. El reto de la inclusión escolar. Educar en Ciudades. Madrid: FIECC-OEI-EuroSocial.

Terigi, F., et. al. (2013). "La educación secundaria obligatoria en la Argentina: entre la expansión del modelo tradicional y las alternativas de baja escala". Revista del IICE, 33. 
REVISTA DE LA ESCUELA DE CIENCIAS DE LA EDUCACIÓN, AÑO 17, NRO. 16, VOL. 2, JULIO A DICIEMBRE DE 2021. PÁGINAS 13-25. ISSN 2362-3349 (EN LÍNEA). POLIITICAS DE INCLUSIÓN EDUCATIVA DE ADOLESCENTES, JÓVENES Y ADULTOS EN LA PROVINCIA DE BUENOS AIRES: TENSIONES EN TORNO A LA SEGMENTACIÓN SOCIO EDUCATIVA Y EL ACCESO AL CONOCIMIENTO. JORGELINA SILVIA SASSERA. NATALIA HERGER

Tiramonti, G. (2008). Una aproximación a la dinámica de la fragmentación del sistema educativo argentino. Especificaciones teóricas y empíricas. Tiramonti, G. y Montes, N. (comp.) La escuela media en debate. Problemas actuales y perspectivas desde la investigación. Buenos Aires: FLACSO- Manantial, 25-38.

Toscano, A. G. \& Diez, M. L. (2012). Principales fortalezas y tensiones. Reflexiones finales. En: Toscano, A. G. (coord). Adolescentes y secundaria obligatoria Finalización de Estudios y Vuelta a la Escuela. Conurbano (Argentina). Buenos Aires: UNICEF.

Toscano, A. G. \& Serial, A. (2012). Presentación descriptiva e implementación del plan. En: Toscano, A. G. (coord). Adolescentes y secundaria obligatoria Finalización de Estudios y Vuelta a la Escuela. Conurbano (Argentina). Buenos Aires: UNICEF.

Tuñón, I. \& Halperín, V. (2009). Desigualdad social en la calidad de la oferta educativa y percepción de la calidad educativa. Primer Congreso de Sociología de la Provincia de Buenos Aires.

Veleda, C. (2005). Efectos segregatorios de la oferta educativa en el Conurbano Bonaerense. Buenos Aires: CIPPEC.

Veleda, C. (2012). La segregación educativa. Entre la fragmentación de las clases medias y la regulación atomizada. Buenos Aires: Editorial Stella, La Crujía.

Wiñar, D. (1981). Educación técnica y estructura social en América Latina. Buenos Aires: UNESCO-CEPAL-PNUD.

Young, M. \& Muller, J. (2016). Curriculum and the specialization of knowledge. Studies in sociology of education. London: Routledge.

Young, M. \& Muller, J. (2013). Tres posibles situaciones educativas para el futuro: lecciones desde la Sociología del Conocimiento. En: Stubrin, A. et al. Simposio Internacional Tensiones entre disciplinas y competencias en el currículum universitario. Santa Fe: Ediciones UNL,197-217.

Young, M. (2009). "What are schools for?”. En: Daniels, H.; Lauder, H. y Porter, J. Knowledge, Values and Educational Policy. Londres: Routledge. 